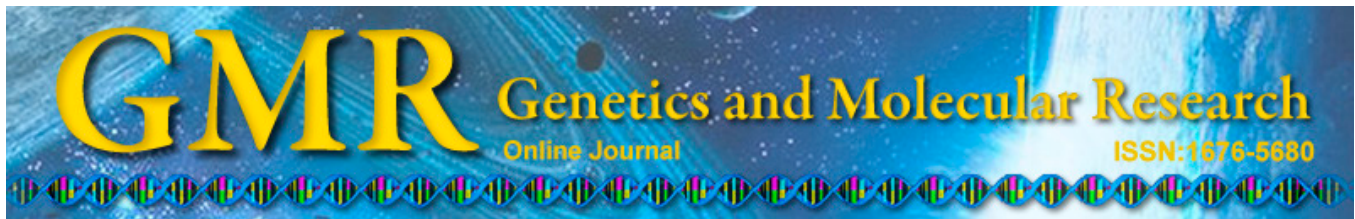

\title{
B-type natriuretic peptide and cirrhosis progression
}

\author{
L.Y. Shi ${ }^{1}$, R. Jin ${ }^{3}$, C.J. Lin ${ }^{1}$, J.S. Wu ${ }^{1}$, X.W. Chen ${ }^{1}$, Z. Yu ${ }^{4}$ and P.C. Zhang ${ }^{2}$ \\ ${ }^{1}$ Department of Gastroenterology and Hepatology, \\ The First Affiliated Hospital of Wenzhou Medical University, Wenzhou, China \\ ${ }^{2}$ Department of General Surgery, \\ The First Affiliated Hospital of Wenzhou Medical University, Wenzhou, China \\ ${ }^{3}$ Department of Epidemiology, \\ The First Affiliated Hospital of Wenzhou Medical University, Wenzhou, China \\ ${ }^{4}$ Department of Surgery, Shanghai Tenth People's Hospital, Shanghai, China
}

Corresponding author: P.C. Zhang

E-mail: peichenzhang_cn@yeah.net

Genet. Mol. Res. 14 (2): 5188-5196 (2015)

Received November 25, 2014

Accepted April 28, 2015

Published May 18, 2015

DOI http://dx.doi.org/10.4238/2015.May.18.9

ABSTRACT. Brain natriuretic peptide (BNP) is used as a marker of
cardiac dysfunction to predict heart failure mortality. The significance
of the prognostic ability of BNP for liver cirrhosis remains unknown,
although the levels of BNP seen in cirrhosis are high. We aimed to
determine whether the BNP level is related to the stage of cirrhosis
and could serve as a prognostic marker of cirrhosis (predict the 1 -year
all-cause mortality). We recruited 92 patients at different stages of
cirrhosis and 81 controls matched by age and gender for this study. At
admission, cardiac physical examination and BNP measurements were
performed. Upon discharge, the 89 patients were followed up for 12
months. The median BNP levels of patients with cirrhosis were 167.0
pg/mL, which were significantly higher than those of the control group
(167.0 vs 34.8 pg/mL, P = 0.001). Serum BNP levels were positively
correlated with the Child score, the grade of esophageal varices, a
history of spontaneous bacterial peritonitis, and the presence of ascites 
and collateral circulation. BNP levels above the median were associated with an increased occurrence of death within 12 months of discharge ( $\log$ rank $\mathrm{P}=0.025$ ), as determined by univariate and multivariate Cox regression analyses. Esophageal varices, large/medium volume ascites, and BNP levels were related to the clinical outcome $(\mathrm{P}=0.034,0.030$, and 0.025 , respectively). Together, these results suggested that serum BNP levels are significantly correlated with the stage of cirrhosis, suggesting that BNP levels might serve as a significant predictor for 1 -year all-cause mortality.

Key words: B-type natriuretic peptide; Cirrhosis; Clinical outcome

\section{INTRODUCTION}

Brain-natriuretic peptide (BNP) is a cardiac hormone secreted from the ventricle in response to pressure or volume overload (Maeda et al., 1998). High BNP levels correlate with left ventricular dysfunction and its prognosis (Chang et al., 2013; Geske et al., 2013). However, high levels of BNP also have been found in congestive states such as heart and renal failure and in chronic liver disease (Passino et al., 2008). Previous studies have shown increased plasma concentrations of BNP in some patients with cirrhosis (Woo et al., 2008; Pimenta et al., 2010), which suggested that cardiac dysfunction might also be present. Recently, several studies have documented that BNP levels were related to the severity of disease in these patients, indicating its predictive value for concomitant cardiac dysfunction and cirrhosis progression (Yildiz et al., 2005). However, the role of BNP as a prognostic marker in that setting has not been fully explored. The aim of our study was to determine the association of BNP levels with the clinical severity of liver cirrhosis (according to the Child-Pugh classification and the associated complications) and to evaluate the significance of BNP as a medium-term prognostic marker in this setting.

\section{MATERIAL AND METHODS}

\section{Subjects}

From April 2008 to September 2010, 92 patients with cirrhosis from the First Affiliated Hospital of Wenzhou Medical University were consecutively included in this study. They consisted of 64 men and 28 women with a median age of 57 years (range, 29-75 years). Diagnoses were based on established clinical, biochemical, and ultra-sonographic criteria. Patients who had diseases affecting BNP levels such as hypertension, diabetes, coronary heart disease, hematopathy, any other major disease, or oral contraceptive use were excluded from the study.

The control group comprised 81 subjects without liver disease, with similar age and gender distributions. All controls had normal arterial blood pressure and no signs of major organic disease. All patients and controls had normal cardiac physical examination, and those who had a chest X-ray demonstrating signs of cardiomegaly were excluded. Electrocardiograms (ECGs) showed normal configurations apart from sporadic extra systoles in some patients. The demographic features of patients, etiological factors, and clinical information were collected. The clinical and biochemical data are summarized in Table 1. This study was 
conducted in accordance with the Declaration of Helsinki and with approval from the Ethics Committee of the First Affiliated Hospital of Wenzhou Medical University. Written informed consent was obtained from all participants.

\section{Biochemical analysis}

Blood was drawn from a forearm vein in the morning following a $12 \mathrm{~h}$ fast and it was collected in standard ethylene diamine tetraacetic acid tubes for BNP measurement, and in the appropriate tubes for other laboratory determinations including liver and renal function tests, blood count, and prothrombin time [PT, as reflected by the international normalized ratio (INR)], performed using standard laboratory automated techniques. The BNP concentration was measured by a sensitive method based on the use of antibodies labeled with a fluorescent dye (BNPTRIAGE, Biosite Diagnostics, San Diego, CA, USA). This is a rapid assay with a measurement time of approximately $15 \mathrm{~min}$ for a single sample, and the test results are calculated automatically. The coefficient of variation for BNP is $10.9 \%$. The measurement range of this assay is $10-5000 \mathrm{pg} / \mathrm{mL}$ and it is designed to have an upper $95 \% \mathrm{CI}$ of $12 \%$ total coefficient of variation.

The stage of cirrhosis was classified according to the Child-Pugh classification, which reflects the severity of the disease. Esophageal varices were detected by endoscopic examination and graded with Paget classification (Sleisenger et al., 1998). Portal-splenic vein diameter was measured using a Doppler-ultrasonography device (ALT-HDI 3500, Philips, USA).

Patients were followed up for 12 months from discharge day to evaluate the incidence and etiology of all-cause mortality. The primary endpoint was all-cause death. Surveillance was made by direct contact with patients or relatives and observation of medical records, by an investigator blinded to the laboratory BNP results.

\section{Statistics analysis}

Data are reported as median (25th-75th percentile) for quantitative variables and as $\mathrm{N}$ (\%) for categorical variables, and was analyzed using the SPSS version 13.0 software (SPSS, Chicago, IL, USA). One way analysis of variance (ANOVA) with Tukey's post hoc pairwise multiple comparison procedure was used. In cases of non-normality, the Kruskal-Wallis test (with Dunn's pairwise comparison) was applied. Correlations were performed by the Pearson regression analysis or in the case of non-linearity by the Spearman-Rank test. Multiple regression analysis was performed to evaluate the relation between BNP and pertinent clinical and biochemical parameters, and with the presence of complications. A stepwise forward modelbuilding approach was employed to evaluate the effects of all variables initially examined. Next, the covariates most probably associated with the survival time in days were investigated by using the Kaplan-Meier (KM) estimator. The significance of the survival was examined by the log-rank test. The association of independent variables with time to clinical outcome was assessed by Cox regression analysis and was expressed as a hazard ratio (HR) and 95\%CI. A $\mathrm{P}<0.05$ was considered to be statistically significant.

\section{RESULTS}

\section{Subject data}

A total of 94 patients with hepatic cirrhosis and 81 controls were recruited for the 
study. Of those, two patients died during hospitalization and were excluded. The median age of the study population was 57 years (range, 29-75 years), including 73 men and 19 women. The causes of cirrhosis were hepatitis B virus infection-associated post-hepatic cirrhosis in 65 patients, hepatitis $\mathrm{C}$ virus infection in one, alcoholism in 12, autoimmune disorder in two, and secondary cirrhosis in four. Among them, 21 patients exhibited ascites, 23 experienced recent gastrointestinal bleeding, 10 had encephalopathy above grade I, and 9 experienced spontaneous bacterial peritonitis. The clinical features of the subjects are shown in Table 1.

Table 1. Clinical and biochemical data of patients with cirrhosis and controls.

\begin{tabular}{|c|c|c|c|}
\hline Characteristics & Cirrhosis group & Control group & $P$ value \\
\hline Age & $57(29-75)$ & $55(27-74)$ & \\
\hline Gender $(\mathrm{F} / \mathrm{M})$ & $19 / 73$ & $13 / 68$ & \\
\hline \multicolumn{4}{|l|}{ Etiology } \\
\hline Viral (N, \%) & $69(0.75)$ & & \\
\hline Alcoholic (N, \%) & $13(0.14)$ & & \\
\hline Other $(\mathrm{N}, \%)$ & $10(0.11)$ & & \\
\hline Child-Pugh class (A/B/C) & $8 / 38 / 46$ & & \\
\hline Child score (points) & $11(7-13)$ & & \\
\hline History of hepatic encephalopathy $(+/-)$ & $10(0.11)$ & & \\
\hline Ascites & $21(0.23)$ & & \\
\hline History of variceal bleeding & $23(0.25)$ & & \\
\hline History of spontaneous bacterial peritonitis & $9(0.098)$ & & \\
\hline Collateral circulation & $21(0.23)$ & & \\
\hline Portal vein diameter $\leq 11 \mathrm{~mm} />11 \mathrm{~mm}$ & $54(0.59)$ & & \\
\hline Splenic vein diameter $\leq 10 \mathrm{~mm} />10 \mathrm{~mm}$ & $46(0.50)$ & & \\
\hline Esophageal varices & $62(0.67)$ & & \\
\hline \multicolumn{4}{|l|}{ Blood analysis } \\
\hline Hemoglobin $(\mathrm{g} / \mathrm{dL})$ & $10.4(4.6-12.8)$ & $14.1(12.5-16.4)$ & 0.001 \\
\hline Platelet count $\left(10^{9} / \mathrm{L}\right)$ & $65(32-143)$ & $261(187-300)$ & 0.001 \\
\hline $\mathrm{BNP}(\mathrm{pg} / \mathrm{mL})$ & $167.0(32.3-652.6)$ & $34.8(10.0-72.4)$ & 0.001 \\
\hline Sodium $(\mathrm{mEq} / \mathrm{L})$ & $130(125-141)$ & $137(135-140)$ & 0.001 \\
\hline Albumin $(\mathrm{g} / \mathrm{dL})$ & $2.47(1.88-2.92)$ & $4.23(3.76-5.21)$ & 0.001 \\
\hline Total bilirubin (mg/dL) & $3.33(2.01-6.63)$ & $0.52(0.44-0.60)$ & 0.001 \\
\hline Creatinine $(\mathrm{mg} / \mathrm{dL})$ & $0.95(0.73-1.20)$ & $1.09(0.87-1.13)$ & 0.389 \\
\hline Prothrombin time (sec) & $18.9(13.2-30.0)$ & $11.5(11.3-13.2)$ & 0.001 \\
\hline
\end{tabular}

$\mathrm{BNP}=$ brain natriuretic peptide.

\section{BNP and Child-Pugh classification}

The BNP levels of patients with hepatic cirrhosis were significantly higher than those in the control group ( $\mathrm{HR}=167.0,95 \% \mathrm{CI}$ : 32.3-652.6 vs $\mathrm{HR}=34.8,95 \% \mathrm{CI}: 10.0-72.4) ; \mathrm{P}=$ 0.001 ). There was also a significant difference among the patients with cirrhosis after classifying them according to Child-Pugh classification, with Child class $\mathrm{B}$ and $\mathrm{C}$ patients exhibiting significantly higher $\mathrm{BNP}$ levels than class A patients $(\mathrm{P}=0.041,0.032$, respectively; Table 2$)$

\section{BNP and complications of cirrhosis}

BNP concentrations were significantly higher in patients who had ascites, esophageal varices, or a history of spontaneous bacterial peritonitis or of hepatic encephalopathy compared with patients without these complications ( $\mathrm{P}<0.05$, respectively). Although the BNP levels of the patients with cirrhosis who had a history of variceal bleeding or portal vein thrombosis were higher than those in the patients without them, there was no statistical difference between the 
BNP levels of the two patient groups $(\mathrm{P}=0.08,0.12$, respectively). The presence of collateral circulation was diagnosed by physical examination or Doppler ultrasonography, and it was observed to also be related to high BNP levels $(\mathrm{P}=0.041)$. Patients with esophageal varices had significantly higher BNP levels than did patients without them $(\mathrm{OR}=163.7,95 \% \mathrm{CI}$ : 60.5-309.5) vs $\mathrm{OR}=116.7,95 \% \mathrm{CI}$ : $51.2-283.1 ; \mathrm{P}=0.045)$. Although BNP levels increased with higher grades of esophageal varices, no significant difference was observed between groups of patients with cirrhosis classified according to variceal size $(\mathrm{P}=0.067)$ in post hoc analyses; BNP levels of patients with varices are shown in Table 3. BNP was not related to the QTc interval $(\mathrm{r}=1.21$, $\mathrm{P}=0.896$ ), although levels in patients with cirrhosis on treatments of beta-blockers and diuretics were significantly higher than those in patients without these treatments (Table 4).

Table 2. BNP concentrations in patients with cirrhosis as related to their Child-Pugh classification.

\begin{tabular}{lrcr}
\hline Group & $\mathrm{N}$ & Median BNP level & P value \\
\hline Controls & 81 & $34.8(10.0-72.4)$ & 0.001 \\
Cirrhosis (total) & 92 & $167.0(32.3-652.6)$ & 0.041 \\
Child class A & 8 & $92.4(32.3-202.1)$ & 0.032 \\
Child class B & 38 & $158.6(58.7-324.1)$ & $224.2(54.2-652.6)$ \\
Child class C & 46 &
\end{tabular}

$\mathrm{BNP}=$ brain natriuretic peptide; $\mathrm{P}<0.001$ : cirrhosis(total) group compare with controls; $\mathrm{P}=0.041$ : child class $\mathrm{B}$ group compare with child class A group; $\mathrm{P}=0.032$ : child class $\mathrm{C}$ group compare with child class $\mathrm{B}$ group.

Table 3. BNP levels according to cirrhosis decompensation component (median).

\begin{tabular}{lccc}
\hline Component & & BNP levels (median) \\
\cline { 2 - 4 } & Present & Absent & P value \\
\hline Ascites & $183.2(35.2-254.2)$ & $126.4(42.1-215.8)$ & 0.045 \\
History of hepatic encephalopathy & $165.1(46.3-278.5)$ & $107.1(38.0-304.9)$ & 0.032 \\
History of variceal bleeding history & $158.1(42.3-183.8)$ & $137.1(51.2-204.7)$ & 0.080 \\
History of spontaneous bacterial peritonitis & $169.3(33.1-312.7)$ & $126.2(52.3-276.2)$ & 0.043 \\
Portal vein thrombosis & $184.2(47.3-365.9)$ & $176.4(33.5-290.6)$ & \\
Esophageal varices & $163.7(60.5-309.5)$ & $116.7(51.2-283.1)$ & \\
Grade 1 & $143.9(55.3-263.7)$ & & \\
Grade 2 & $165.6(43.8-249.5)$ & & \\
Grade 3 & $174.7(60.5-304.8)$ & & \\
Collateral circulation & $154.3(38.5-326.3)$ & $103.4(40.2-255.8)$ & \\
Portal vein diameter $\leq 11 \mathrm{~mm} />11 \mathrm{~mm}$ & $156.7(40.6-287.4)$ & $110.9(45.2-278.8)$ & 0.041 \\
\hline
\end{tabular}

$\mathrm{BNP}=$ brain natriuretic peptide.

Table 4. Comparision of patients with and without drug treatment for BNP levels.

\begin{tabular}{llll}
\hline Drug & N & Median BNP levels & P \\
\hline Beta blocker (+) & 32 & $219.7(102.3-542.7)$ & 0.031 \\
Beta blocker (-) & 60 & $158.3(63.2-215,4)$ & 0.023 \\
Diuretic (+) & 24 & $272.9(118.9-487.2)$ & $154.5(45.4-258.7)$ \\
Diuretic (-) & 69 & 1 & \\
\hline
\end{tabular}

$\mathrm{BNP}=$ brain natriuretic peptide.

\section{BNP and prognosis}

During the follow-up period, 11 patients died (six of hemorrhage, two of portosystemic encephalopathy, two of infections, and one of liver failure) and two were lost to follow-up. In 
univariate Cox regression analysis, more than one factor was shown to be related to outcome, including age, BNP level, esophageal varices, total bilirubin, hepatic encephalopathy history, and large/medium ascites volume (Table 5); in contrast, in multivariate analysis, only three factors were flagged as independent predictors of survival at 12 months: BNP levels, esophageal varices, and large/medium volume ascites (Table 6). BNP levels dichotomized according to the median $(167 \mathrm{pg} / \mathrm{mL})$ were associated with the occurrence of all-cause mortality at 12 months of followup, as evaluated by Kaplan-Meier analysis ( $\log$ rank test $\mathrm{P}=0.023)$ (Figure 1$)$.

Table 5. Univariate and multivariate Cox regression model for predictors of all-cause mortality within twelve months after discharge in patients with cirrhosis.

\begin{tabular}{|c|c|c|c|}
\hline Variable & Hazard ratio & $95 \%$ confidence interval & $P$ value \\
\hline Age $>55$ years & 2.23 & $0.98-4.97$ & 0.049 \\
\hline Large/medium volume ascites ( $v s$ small/none) & 2.46 & $0.87-3.23$ & 0.047 \\
\hline History of hepatic encephalopathy & 2.09 & $0.97-4.25$ & 0.052 \\
\hline History of variceal bleeding & 2.02 & $0.74-3.95$ & 0.147 \\
\hline History of spontaneous bacterial peritonitis & 1.86 & $0.65-2.96$ & 0.230 \\
\hline Portal vein thrombosis & 1.62 & $0.52-3.04$ & 0.539 \\
\hline Esophageal varices & 2.78 & $1.04-6.13$ & 0.040 \\
\hline Collateral circulation & 2.04 & $0.96-4.31$ & 0.67 \\
\hline $\mathrm{BNP}>167.0 \mathrm{pg} / \mathrm{mL}$ & 2.95 & $1.13-7.24$ & 0.032 \\
\hline Haemoglobin $>10.4 \mathrm{~g} / \mathrm{dL}$ & 1.36 & $0.84-3.54$ & 0.79 \\
\hline Platelet count $>65$ & 1.29 & $0.72-3.02$ & 0.652 \\
\hline Albumin $>2.47 \mathrm{~g} / \mathrm{dL}$ & 1.24 & $0.43-2.92$ & 0.683 \\
\hline Serum sodium $>130 \mathrm{mEq} / \mathrm{L}$ & 1.33 & $0.52-3.01$ & 0.702 \\
\hline Total bilirubin $>3.33 \mathrm{~g} / \mathrm{dL}$ & 2.01 & $0.65-4.28$ & 0.067 \\
\hline Prothrombin time $>18.9 \mathrm{~s}$ & 1.92 & $0.98-3.16$ & 0.475 \\
\hline Child-Pugh score $>11$ points & 2.62 & $0.96-4.88$ & 0.041 \\
\hline $\mathrm{QTc}>0.420$ & 1.27 & $0.45-2.71$ & 0.896 \\
\hline
\end{tabular}

$\mathrm{BNP}=$ brain natriuretic peptide; $\mathrm{QTc}=$ corrected QT interval.

Table 6. Multivariate Cox regression model for predictors of all-cause mortality within twelve months after discharge in patients with cirrhosis.

\begin{tabular}{lccc}
\hline Predictors & Hazard ratio & 95\% confidence interval & P value \\
\hline Esophageal varices & 2.64 & $1.13-3.04$ & 0.034 \\
Large/medium volume ascites ( $v s$ small/none) & 2.35 & $1.47-3.12$ & 0.030 \\
BNP $>167 \mathrm{pg} / \mathrm{mL}$ & 2.92 & $1.75-4.08$ & 0.025 \\
\hline
\end{tabular}

$\mathrm{BNP}=$ brain natriuretic peptide.

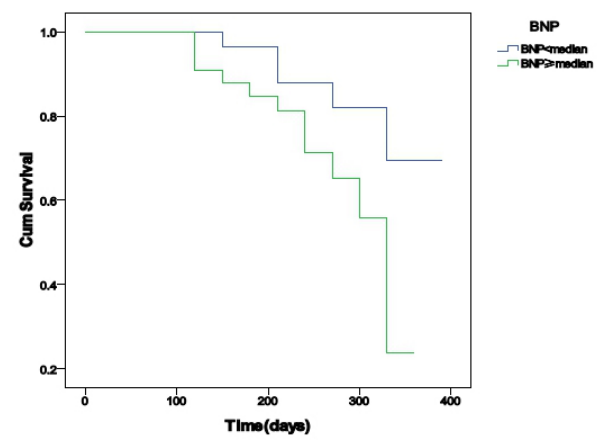

Figure 1. Survival of patients with decompensated cirrhosis according to their BNP levels. The BNP levels at hospital admission of patients with decompensated cirrhosis dichotomized according to the median $(167 \mathrm{pg} / \mathrm{mL})$ were associated with the occurrence of all-cause mortality at twelve months of follow-up, as evaluated by KaplanMeier analysis $(\log$ rank test $\mathrm{P}=0.025)$. 


\section{DISCUSSION}

Our results showed that: first, serum BNP levels were significantly increased in patients with cirrhosis compared with controls; second, BNP concentrations were related to the severity of cirrhosis according to Child-Pugh classification and the decompensation component of cirrhosis; and third, BNP levels were associated with medium-term survival, implying its potential utility as a prognostic predictor in cirrhosis.

Several earlier studies have shown high BNP levels in chronic liver disease, and our study also confirms previous findings that the BNP levels of healthy individuals and of patients with cirrhosis differ. On the other hand, we found an association between BNP levels and the Child-Pugh score, wherein Child C and B groups had higher BNP levels than did the Child A group, which in turn had higher BNP levels than did the control group in our study. These findings are supported by those of Henriksen et al. (2003), and indicate that BNP might be an early indicator of cirrhosis in patients with hepatocellular diseases and might also be related to the severity of cirrhosis.

According to the results of this study, the existence of ascites, collateral circulation, a history of spontaneous bacterial peritonitis, and esophageal varices were related to increased BNP levels. Although there was a lack of statistical significance, higher variceal grades tended to be present together with higher BNP levels. These findings are indicators of high portal vein pressure, and cirrhotic patients with portal hypertension had significantly higher BNP levels than did patients without portal hypertension according to the results of this study. These findings indicate that BNP might be also a prediction tool to follow the clinical progress of patients with cirrhosis. BNP might also be used for pursuing the responses to the treatment given to these patients. However, further studies are needed in order to reveal the relationship between BNP and treatment modalities in patients with cirrhosis.

We also discovered that the BNP levels of patients on treatments with beta-blockers and diuretics were significantly higher than those of patients without treatment, which is not associated with clinical experience. de Lemos et al. (2003) and some others have advocated that the net effect of beta-blockers and diuretics is often to reduce BNP concentrations (Park et al., 2010; Luchner et al., 2013). In cirrhotic cardiomyopathy, beta adrenergic receptor and plasma membrane dysfunction are observed in addition to an increase in intracardiac volume; therefore, diuretics and beta-blockers likely decrease BNP levels in patients with decompensated heart failure (Johnson et al., 2002) by improving hemodynamic parameters and the left ventricular ejection fraction (Møller and Henriksen, 2002; Fujimura et al., 2009), thus reducing intracardiac pressure and sodium retention and consequently decreasing the secretion of BNP. However, we draw the opposite conclusion in this study from our results than did Yilmaz et al. (2010), who surmised that these findings might be a result of a selection bias, because the probable underlying cause of increased levels of BNP in patients using beta-blockers and diuretics might be that these patients were more likely to suffer from volume overload, and that these medications are usually given in these patients. However, the underlying cause of the increased BNP levels seen in patients with cirrhosis is still unknown. Several factors can be assumed to be responsible: 1) altered hepatic (or renal) degradation; 2) increased heart secretion as a result of hyperkinetic circulation and/or latent subclinical heart failure-cirrhotic cardiomyopathy; or 3) the consequence of resistance to natriuretic peptides. Most studies have reported that increased BNP levels in patients with cirrhosis were associated with cardiac systolic function. Henriksen et al. (2003) reported that BNP levels were related to markers of cardiac dysfunction, such as QT interval, heart rate, and plasma volume. In contrast to previ- 
ous studies (Padillo et al., 2010; Bernal et al., 2012), there was no relationship between BNP concentration and QTc interval identified in our research. It has been reported that a level of BNP over $500 \mathrm{pg} / \mathrm{mL}$ is diagnostic for the congestive heart failure presented and diagnosed by a lower ejection fraction on echocardiographic examination (Buğdayc1 and Sökmen, 2012). As the BNP levels in our study were almost below $500 \mathrm{pg} / \mathrm{mL}$, this might suggest that the increased BNP levels were not associated with any cardiac dysfunction in our patients; also, because we did not find any relationship between the serum sodium and BNP levels, the elevated BNP levels might be instead associated with liver failure. In this study, we found that a history of hepatic encephalopathy and spontaneous bacterial peritonitis were related to increased BNP levels in patients with cirrhosis. Ascites and hepatic encephalopathy are part of the parameters of the Child-Pugh classification that indicate the severity of liver disease; the results of our study suggested that the presence of these were related to increased BNP levels.

Decompensated cirrhosis is a condition with grim prognosis. While death in patients with cirrhosis generally occurs because of end-stage liver failure or portal hypertension, in a multivariate Cox regression mode, ascites, esophageal varices, and BNP levels were all independent predictors of outcome. Ascites confers a poor prognosis because of the associated development of spontaneous bacterial peritonitis and hepatorenal syndrome. We found that BNP was an independent predictor in multivariate Cox regression analysis, similar to previous results (Pimenta et al., 2010). BNP concentrations in patients with cirrhosis have been reported to be an independent predictor of medium-term (6-month) survival in hospitalized patients with decompensated cirrhosis, suggesting its utility in risk stratification of patients with advanced cirrhosis (Pimenta et al., 2010); this finder also was supported by the results of Saner et al. (2011). This finding was not unexpected because, as mentioned above, increased BNP concentrations were found to be associated with the decompensation components of cirrhosis such as hepatic encephalopathy, esophageal varices, and ascites. As is well recognized, esophageal variceal bleeding is a major cause of death in cirrhosis (Krishnan et al., 2012), and hepatic encephalopathy is also a leading cause of death. BNP is a known marker of cardiac systolic function; however, as was also the case with our patients, BNP levels can be influenced by other hemodynamic variables as well as non-cardiac-related parameters such as age, renal function, hemoglobin, and even albumin, a marker of liver failure (Huh et al., 2012). Taken together, therefore, it appears plausible that BNP levels might serve as a mirror of this complex milieu, and might be of prognostic usefulness in patients with cirrhosis.

In conclusion, serum BNP levels were found to correlate significantly with the stage of cirrhosis in our study. BNP levels also were seen to be a significant predictor for 1-year all-cause mortality, indicating that measurements of BNP might be a simple and valuable diagnostic test for the prediction of the mortality of patients with decompensated cirrhosis.

\section{Conflicts of interest}

The authors declare no conflict of interest.

\section{ACKNOWLEDGMENTS}

Research supported by the Wenzhou Municipal Science and Technology Bureau (\#Y20120209) and the Medical Supporting Discipline Construction Program of Zhejiang Province (\#11-ZC24). 


\section{REFERENCES}

Bernal V, Pascaul I, Lanas A, Esquivias P, et al. (2012). Cardiac function and aminoterminal pro-brain natriuretic peptide levels in liver-transplanted cirrhotic patients. Clin. Transplant. 26: 111-116.

Buğdayc1 MS and Sökmen M (2012). B-type natriuretic peptide and cirrhosis: is there any relation? Turk. J. Gastroenterol. 23: 824.

Chang HR, Hsieh JC, Hsu BG, Wang LY, et al. (2013). Inverse association of N-terminal pro-B-type natriuretic peptide with metabolic syndrome in patients with congestive heart failure. PLoS One 8: e79096.

de Lemos JA, McGuire DK and Drazner MH (2003). B-type natriuretic peptide in cardiovascular disease. Lancet 362: 316-322.

Fujimura M, Akaike M, Iwase T, Yoshida S, et al. (2009). Decrease in plasma brain natriuretic peptide level in the early phase after the start of carvedilol therapy is a novel predictor of long-term outcome in patients with chronic heart failure. Acta Cardiol. 64: 589-595.

Geske JB, McKie PM, Ommen SR and Sorajja P (2013). B-type natriuretic peptide and survival in hypertrophic cardiomyopathy. J. Am. Coll. Cardiol. 61: 2456-2460.

Henriksen JH, Gøtze JP, Fuglsang S, Christensen E, et al. (2003). Increased circulating pro-brain natriuretic peptide (proBNP) and brain natriuretic peptide (BNP) in patients with cirrhosis: relation to cardiovascular dysfunction and severity of disease. Gut 52: 1511-1517.

Huh IY, Kim YK, Shin WJ, Park SE, et al. (2012). Increased B-type natriuretic peptide during liver transplantation: relationship to invasively measured hemodynamic parameters. Transplant Proc. 44: 1318-1322.

Johnson W, Omland T, Hall C, Lucas C, et al. (2002). Neurohormonal activation rapidly decreases after intravenous therapy with diuretics and vasodilators for class IV heart failure. J. Am. Coll. Cardiol. 39: 1623-1629.

Krishnan A, Srinivasan V and Venkataraman J (2012). Variceal recurrence, rebleeding rates and alterations in clinical and laboratory parameters following post-variceal obliteration using endoscopic sclerotherapy. J. Dig. Dis. 13: 596-600.

Luchner A, Behrens G, Stritzke J, Markus M, et al. (2013). Long-term pattern of brain natriuretic peptide and N-terminal pro brain natriuretic peptide and its determinants in the general population: contribution of age, gender, and cardiac and extra-cardiac factors. Eur. J. Heart Fail. 15: 859-867.

Maeda K, Tsutamoto T, Wada A, Hisanaga T, et al. (1998). Plasma brain natriuretic peptide as a biochemical marker of high left ventricular end-diastolic pressure in patients with symptomatic left ventricular dysfunction. Am. Heart J. 135: 825-832.

Møller S and Henriksen JH (2002). Cirrhotic cardiomyopathy: a pathophysiological review of circulatory dysfunction in liver disease. Heart 87: 9-15.

Padillo J, Rioja P, Muñoz-Villanueva MC, Vallejo JA, et al. (2010). BNP as marker of heart dysfunction in patients with liver cirrhosis. Eur. J. Gastroenterol. Hepatol. 22: 1331-1336.

Park HJ, Baek SH, Jang SW, Kim DB, et al. (2010). Direct comparison of B-type natriuretic peptide and N-terminal proBNP for assessment of cardiac function in a large population of symptomatic patients. Int. J. Cardiol. 140: 336-343.

Passino C, Poletti R, Fontana M, Vergaro G, et al. (2008). Clinical relevance of non-cardiac determinants of natriuretic peptide levels. Clin. Chem. Lab. Med. 46: 1515-1523.

Pimenta J, Paulo C, Gomes A, Silva S, et al. (2010). B-type natriuretic peptide is related to cardiac function and prognosis in hospitalized patients with decompensated cirrhosis. Liver Int. 30: 1059-1066.

Saner FH, Neumann T, Canbay A, Treckmann JW, et al. (2011). High brain-natriuretic peptide level predicts cirrhotic cardiomyopathy in liver transplant patients. Transpl. Int. 24: 425-432.

Sleisenger MH, Fordtran JS, Feldman M and Scharschmitd B (1998). Sleisenger and Fordtran's gastrointestinal and liver disease. 6th edn. (Feldman M, Sleisenger MH and Scharschmidt BF, eds.).WB Saunders, Philadelphia, pp. 12841309.

Woo JJ, Koh YY, Kim HJ, Chung JW, et al. (2008). N-terminal pro B-type natriuretic peptide and the evaluation of cardiac dysfunction and severity of disease in cirrhotic patients. Yonsei Med. J. 49: 625-631.

Yildiz R, Yildirim B, Karincaoglu MJ, Harputiuoglu M, et al. (2005). Brain natriuretic peptide and severity of disease in non-alcoholic cirrhotic patients. J. Gastroenterol. Hepatol. 20: 1115-1120.

Yilmaz VT, Eken C, Avci AB, Duman A, et al. (2010). Relationship of increased serum brain natriuretic peptide levels with hepatic failure, portal hypertension and treatment in patients with cirrhosis. Turk. J. Gastroenterol. 21: 381-386. 